\title{
Venous thromboembolism and COVID-19: a single center experience from an academic tertiary referral hospital of Northern Italy
}

\author{
Federica Melazzini ${ }^{1}$ (D) Marta Colaneri ${ }^{2} \cdot$ Federica Fumoso $^{1} \cdot$ Giulia Freddi $^{1} \cdot$ Marco Vincenzo Lenti $^{1}$. \\ Teresa Chiara Pieri ${ }^{2}$. Davide Piloni ${ }^{3}$. Patrizia Noris ${ }^{1}$. Carla Pieresca ${ }^{1} \cdot$ Paola Stefania Preti ${ }^{1} \cdot$ Mariaconcetta Russo $^{1}$. \\ Angelo Corsico ${ }^{3}$. Guido Tavazzi ${ }^{4}$. Fausto Baldanti ${ }^{5}$. Antonio Triarico ${ }^{6}$. Francesco Mojoli ${ }^{4} \cdot$ Raffaele Bruno $^{2}$. \\ Antonio Di Sabatino ${ }^{1}$ on behalf of the San Matteo Pavia COVID-19 Task Force
}

Received: 17 July 2020 / Accepted: 22 October 2020 / Published online: 8 November 2020

(c) The Author(s) 2020

\begin{abstract}
Preliminary evidence supports the notion that COVID-19 patients may have an increased susceptibility to develop venous thromboembolism (VTE). However, the magnitude of this association still needs to be defined. Furthermore, clinical predictors of thrombogenesis, and the relationship with the inflammatory status are currently unknown. On this basis, we conducted a retrospective, observational study on 259 consecutive COVID-19 patients admitted to an academic tertiary referral hospital in Northern Italy between March 19th and April 6th, 2020. Records of COVID-19 patients with a definite VTE event were reviewed for demographic information, co-morbidities, risk factors for VTE, laboratory tests, and anticoagulation treatment. Twenty-five cases among 259 COVID-19 patients developed VTE (9.6\%), all of them having a Padua score $>4$, although being under standard anticoagulation prophylaxis since hospital admission. In the VTE subcohort, we found a significant positive correlation between platelet count (PLT) and either C reactive protein (CRP) $(p<0.0001)$ or lactate dehydrogenase (LDH) $(p=0.0013)$, while a significant inverse correlation was observed between PLT and mean platelet volume $(p<0.0001)$. Platelet-to-lymphocyte ratio significantly correlated with CRP $(p<0.0001)$. The majority of VTE patients was male and younger compared to non-VTE patients ( $p=0.002$ and $p=0.005$, respectively). No significant difference was found in D-dimer levels between VTE and non VTE patients, while significantly higher levels of LDH $(p=0.04)$ and IL-6 $(p=0.04)$ were observed in VTE patients in comparison to non-VTE patients. In conclusion, our findings showed a quite high prevalence of VTE in COVID-19 patients. Raised inflammatory indexes and increased serum levels of pro-inflammatory cytokines should raise the clinical suspicion of VTE.
\end{abstract}

Keywords Anticoagulants $\cdot$ Pulmonary embolism $\cdot$ SARS-CoV-2 $\cdot$ Thrombosis

The members of San Matteo Pavia COVID-19 Task Force are listed in Acknowledgements section.

Electronic supplementary material The online version of this article (https://doi.org/10.1007/s11739-020-02550-6) contains supplementary material, which is available to authorized users.

Antonio Di Sabatino

a.disabatino@smatteo.pv.it

1 Department of Internal Medicine, San Matteo Hospital Foundation, University of Pavia, Pavia, Italy

2 Department of Infectious Disease, San Matteo Hospital Foundation, University of Pavia, Pavia, Italy

3 Department of Respiratory Disease, San Matteo Hospital Foundation, University of Pavia, Pavia, Italy

\section{Introduction}

Coronavirus disease 2019 (COVID-19) is caused by severe acute respiratory syndrome coronavirus 2 (SARS-CoV-2), which is responsible for acute respiratory distress syndrome

4 Department of Intensive Care, San Matteo Hospital Foundation, University of Pavia, Pavia, Italy

5 Molecular Virology Unit, Microbiology and Virology Department, San Matteo Hospital Foundation, University of Pavia, Pavia, Italy

6 Chief Medical Direction, San Matteo Hospital Foundation, Pavia, Italy 
in a significant proportion of affected patients $(27-31 \%)[1$, 2].

The clinical spectrum of SARS-CoV-2 infection appears to be wide, encompassing asymptomatic infection, mild flulike syndrome, and severe viral pneumonia with respiratory failure and superimposed bacterial infections which may be fatal [3-5].

Venous thromboembolism (VTE) includes deep vein thrombosis (DVT), pulmonary embolism (PE) and portal vein thrombosis. The association between VTE and COVID-19 was described for the first time by Zhou et al. [6], who identified thrombotic events in $2.9 \%$ of a cohort of COVID-19 patients. In previous Asian series, thromboembolic events have been reported in roughly one fourth of COVID-19 patients admitted to the intensive care unit, and these findings correlated with a poor prognosis [7]. A number of pathogenic mechanisms have been hypothesized for VTE in COVID-19 patients, including active inflammation, immobilization and intensive care treatments, but the limited evidence available in the literature does not allow to estimate the relative contribution of each of the abovementioned factors [8].

Little is known regarding the risk of VTE in non-Asian countries, that are known to have a higher incidence of VTE in comparison to Asian countries [9]. Recent publications reported a high rate of VTE and arterial thrombotic events especially in critically ill patients (10-36\% depending on the analyzed cohort), also in Occidental cohorts of COVID-19 subjects [10-15].

Starting from these premises, we here aimed to define VTE rates and types, not considering peripheral and central catheter-related thrombosis, among a cohort of COVID-19 patients during their hospital stay at the San Matteo Hospital Foundation (Pavia, Northern Italy).

\section{Methods}

This is a single-center, retrospective, observational study. We extracted data from medical records of all 259 consecutive patients with a diagnosis of COVID-19 admitted to the Departments of Internal Medicine, Infectious Disease, Intensive Care, and Respiratory Disease of the San Matteo Hospital Foundation (Pavia, Northern Italy), between March 19th and April 6th, 2020. The enrolment was limited to this time lapse as exhaustive data were available only for patients enrolled during this period. All patients had a confirmed diagnosis of COVID-19 by Real Time Polymerase Chain Reaction (RT-PCR) in respiratory samples (see Supplementary materials).

We reviewed records of all 259 COVID-19 patients for demographic information, co-morbidities, risk factors for
VTE according to the Padua prediction score [16], laboratory tests and anticoagulation treatment at the time of hospital admission.

DVT and PE were diagnosed through ultrasound and CTscan or CT-angiography, respectively. In particular, every patient with clinical suspect of VTE or worsening respiratory function, underwent four-points US or CT-scan. The regions tested included: the common femoral vein at the level of inguinal crease, the superficial femoral vein superior to the adductor canal, the popliteal vein in the popliteal fossa and the great saphenous vein. The remaining patients were tested for routine laboratory tests if not daily, at least every 2-3 days. Symptomatic patients were defined as follows: worsening $\mathrm{PaO}_{2} / \mathrm{FiO}_{2}$ despite optimized therapy, signs and symptoms of DVT (i.e., pain, swelling, warmth), increase of D-dimer. No peripheral and central catheter-related DVTs were included in the study.

We also examined the correlation between DVT and selected inflammatory markers, including C-reactive protein (CRP), interleukin (IL)-6, mean platelet volume (MPV), neutrophil-to-lymphocyte ratio (NLR), and platelet-to-lymphocyte ratio (PLR).

The study was performed as a clinical audit using routinely collected clinical data and as such is exempt from the need to require written informed consent. The study was approved by the local ethics committee (San Matteo Hospital Foundation) on March 13th 2020.

The results of this study are reported according to the STrengthening the Reporting of OBservational studies in Epidemiology (STROBE) recommendations [17].

\section{Statistical analyses}

Given the observational nature of the study, sample size was not calculated a priori. Continuous variables were expressed as median and range and compared with the Mann-Whitney $U$ test. Categorical variables were described as number (\%), and proportions for categorical variables were compared using the Fisher exact test. Spearman correlation coefficient was calculated for relevant laboratory parameters. When variables were not available for some patients, these were excluded for percentage calculation. A two-sided $\alpha$ of $<0.05$ was considered statistically significant. Statistical analyses were performed using MedCalc (Belgium, version 2020).

\section{Results}

A total of 259 cases of definite COVID-19 patients were included in the registry between March 19th and April 6th 2020. Demographic characteristics, medical history and laboratory parameters of all patients included in the study 
Table 1 Demographic characteristics, medical history and laboratory results of the two whole cohort of COVID-19 patients enrolled in the study, and of the subcohorts of patients with and without VTE at the time of hospital admission

\begin{tabular}{|c|c|c|c|c|}
\hline & $\begin{array}{l}\text { All patients } \\
(n=259)\end{array}$ & $\begin{array}{l}\text { VTE } \\
(n=25)\end{array}$ & $\begin{array}{l}\text { Non-VTE } \\
(n=234)\end{array}$ & $p$ value \\
\hline Median age, years (range) & $70(25-97)$ & $62(44-84)$ & $70(25-97)$ & 0.005 \\
\hline \multicolumn{5}{|l|}{ Sex } \\
\hline Female & $66(25-94)$ & $63(44-84)$ & $69(25-94)$ & 0.006 \\
\hline n. $(\%)$ & $(32)$ & $(24)$ & $(32)$ & \\
\hline Male & 74 (27-97) & $62(51-79)$ & 79 (27-97) & 0.002 \\
\hline n. $(\%)$ & $(68)$ & (76) & $(68)$ & \\
\hline \multicolumn{5}{|l|}{ Padua score } \\
\hline$>4$ & 226 & 25 & 202 & 0.4 \\
\hline n. $(\%)$ & $(87)$ & $(100)$ & $(86)$ & \\
\hline$\leq 4$ & 45 & 0 & 45 & $<0.0001$ \\
\hline n. $(\%)$ & (13) & $(0)$ & $(14)$ & \\
\hline \multicolumn{5}{|l|}{ Ventilation } \\
\hline Non-invasive ventilation & 205 & 16 & 189 & 0.2 \\
\hline n. $(\%)$ & (79) & $(64)$ & $(81)$ & \\
\hline Invasive mechanical ventilation & 54 & 9 & 45 & 0.29 \\
\hline n. $(\%)$ & $(21)$ & $(36)$ & (19) & \\
\hline \multicolumn{5}{|l|}{ Anticoagulant prophylactic therapy } \\
\hline Enoxaparin & 206 & 14 & 192 & 0.07 \\
\hline n. $(\%)$ & (79) & $(56)$ & $(82)$ & \\
\hline Fondaparinux & 3 & 2 & 1 & 0.06 \\
\hline n. $(\%)$ & (1) & $(8)$ & (4) & \\
\hline Calcic heparin & 30 & 7 & 23 & 0.03 \\
\hline n. $(\%)$ & $(12)$ & $(28)$ & $(10)$ & \\
\hline \multicolumn{5}{|l|}{ Department } \\
\hline Subintensive/intermediate care & 205 & 16 & 189 & 0.07 \\
\hline n. $(\%)$ & $(79)$ & $(64)$ & $(81)$ & \\
\hline Intensive care unit & 54 & 9 & 45 & 0.04 \\
\hline$n .(\%)$ & $(21)$ & $(36)$ & $(19)$ & \\
\hline \multicolumn{5}{|l|}{ Laboratory parameters } \\
\hline Haemoglobin (g/dl), median (range) & $12.5(6.8-17.7)$ & $13.4(8.9-15.5)$ & $12.4(17.7-6.8)$ & 0.08 \\
\hline Leukocytes $(\mathrm{n} / \mu \mathrm{l})$, median (range) & $7.1(0.2-91.3)$ & $9.2(3.1-17.7)$ & $6.7(0.2-91.3)$ & 0.2 \\
\hline Lymphocytes $(\mathrm{n} / \mu \mathrm{l})$, median (range) & $0.7(0.02-86.2)$ & $0.7(0.2-4.9)$ & $0.7(0.1-86.2)$ & 0.39 \\
\hline NLR, median (range) & $8.2(0.1-390.5)$ & $13.7(2.3-25.1)$ & $8.2(0.1-390.5)$ & 0.5 \\
\hline Platelets $\left(\times 10^{3} / \mu \mathrm{l}\right)$, median (range) & $193(12-768)$ & $184(80-598)$ & $197(12-768)$ & 0.7 \\
\hline LDH (mU/ml), median (range) & $369(142-4641)$ & $590(184-4641)$ & $369(142-2578)$ & 0.04 \\
\hline C-reactive protein (mg/dl), median (range) & $12.3(0.2-47.4)$ & $22(3.5-47.4)$ & $12.2(0.2-42.5)$ & 0.05 \\
\hline Procalcitonine (ng/ml), median (range) & $0.3(0-206)$ & $0.4(0-56.4)$ & $0.3(0-206)$ & 0.9 \\
\hline D-Dimer $(\mu \mathrm{g} / \mathrm{l})$, median (range) & $5181(555-35,000)$ & $15,350(3824-35,000)$ & $3229(555-35,000)$ & 0.09 \\
\hline Interleukin-6 (mg/dl), median (range) & $114.9(5.7-386.4)$ & $163.5(24.2-386.4)$ & $66.2(5.7-215.3)$ & 0.04 \\
\hline
\end{tabular}

Statistically significant p-values are indicated in bold

$L D H$ lactate dehydrogenase, NLR neutrophil-to-lymphocyte ratio, $P L R$ platelet-to-lymphocyte ratio, VTE venous thromboembolism

are reported in Table 1. The median age of the patients was 70 years (range $25-97$ ), $32 \%$ of the total were female (median age 66, range 25-94), 68\% were male with a median age of 74 (range 27-97).
Twenty-five patients turned out to be complicated by VTE (9.6\%), of which 21 had DVT, four had PE, and one among them also had portal vein thrombosis. All patients with VTE had a Padua score $>4$. We did not identify any incidental diagnosis of VTE, because asymptomatic patients did not 
undergo diagnostic work-up. Of the 259 enrolled patients, $239(92.3 \%)$ received thromboprophylaxis. Among them, 24 (10.4\%) developed VTE.

Twenty-two out of the 25 VTE cohort patients underwent prophylactic therapy with low molecular weight heparin (LWMH) or calcic heparin. Three patients of the VTE-group had clinical evidence of lower DVT by the time of hospital admission, hence anticoagulant therapy was immediately started. The median time lapse between hospital admission and VTE onset was 5.0 days (range $0-5$ days). The median time lapse between onset of COVID-19-related symptoms and VTE was 15.0 days (range 10-30 days). DVT was the most frequent event, distributed among upper extremities and lower limb, being upper DVT more prevalent. Of note, the prevalence of VTE in ICU patients was higher in respect to sub intensive/intermediate care patients departments ( $17 \%$ and $8 \%$, respectively; $p=0.02)$. There was a significant correlation between platelet parameters and inflammation. In particular, platelet count directly correlated with either CRP $\left(p<0.0001, r_{\mathrm{s}}=0.13392\right)$ or LDH $\left(p=0.0013, r_{\mathrm{s}}=0.05438\right)$, and it inversely correlated with MPV $\left(p<0.0001, r_{\mathrm{s}}=-0.75608\right)$. MPV significantly correlated in an inverse manner with either CRP $(p<0.0001$, $\left.r_{\mathrm{s}}=-0.01657\right)$ or LDH $\left(p<0.0001, r_{\mathrm{s}}=0.16386\right)$. PLR significantly correlated directly with CRP $(p<0.0001$, $\left.r_{\mathrm{s}}=0.35769\right)$. D-dimer correlated directly with PLT $\left(p=0.006, r_{\mathrm{s}}=0.03036\right), \mathrm{LDH}\left(p=0.007, r_{\mathrm{s}}=0.33395\right)$ and inversely with MPV $\left(p<0.0001, r_{\mathrm{s}}=0.5161\right)$ and lymphocyte $\left(p<0.0001, r_{\mathrm{s}}=-0.0308\right)$. Of note, in the non-VTE group a correlation between PLR and CRP was not observed $\left(p=0.9, r_{\mathrm{s}}=0.20984\right)$ (Table 2).

Furthermore, $76 \%$ of VTE patients were male and the median age at admission was 62 (range 44-84), while in the non-VTE group patients were significantly older (median 70 , range $25-97$ ), and the male population was less represented. No differences were found between VTE and nonVTE cohorts in terms of anticoagulant prophylactic therapy.

There was no significant difference in D-dimer levels between the VTE and non-VTE patients $(p=0.09)$. LDH was significantly higher in VTE-group compared to the nonVTE group (median 590, range 184-4641 mU/ml). Serum levels of IL-6 were significantly increased in VTE compared to non-VTE patients $(p=0.04)$. All the other parameters did not significantly differ between VTE and non-VTE patients.

Supplementary Table 1 shows characteristics and differences between VTE patients admitted to intensive care unit (ICU) and VTE general ward patients.

\section{Discussion}

Our findings showed a high prevalence (9.6\%) of VTE in a non-Asian cohort of COVID-19 patients, which is higher when compared to the prevalence of VTE in patients hospitalized for other causes $(1.2-2.0 \%)$ [18, 19]. Surprisingly, this high rate of VTE was encountered despite prophylactic anticoagulation therapy in almost all patients. In fact, of all the patients who received thromboprophylaxis, $10.4 \%$ developed VTE, which is a higher rate compared to the standard of $0-2 \%$ of symptomatic VTE during enoxaparin prophylaxis. In the subcohort of ICU patients, VTE rate was $16.6 \%$, which is rather comparable to the literature-reported rate for these critically ill patients (10-50\%, if VTE is screened). Indeed, the VTE rate in our cohort is lower than that from some other recent reports $[10,12]$. This might reflect the patients' setting, since a minority of our patients was managed in the ICU and underwent chest CT-scan.

Surprisingly, 13 out of 25 VTE patients (52\%) presented with upper extremities DVT. Of note, 8 of these were ICU patients (62\%), while the remaining $5(38 \%)$ were general ward patients and all of them received supportive ventilation through c-PAP. These findings support the notion that intensive care expose critically ill patients to a higher risk of developing DVT. In fact, critically ill patients are at high risk of VTE as they are susceptible to both general risks factors of VTE as well as those specific to ICU patients, such as sedation, immobilization, and use of vasopressors [20]. Furthermore, peripheral lines and peripherally inserted central catheters (PICCs) for intravenous therapy, could have contributed to the thrombotic process, particularly in an inflammatory state like the COVID-19 related one.

The relationship between viral pneumonia and predisposition to VTE has been well described [21, 22]. This phenomenon is the result of interaction between activated leukocytes and cellular adhesion molecules on the vein wall [23-30]. Inflammatory mechanisms up-regulate procoagulant factors, down-regulate natural anticoagulants and inhibit fibrinolytic activity [31]. In particular, as Marongiu et al. assert, the massive endothelial damage and activation of coagulation cascade is local [32], leading to pulmonary microvessels thrombosis [33]. Therefore, our study supports the notion that VTE correlates with an inflammatory burst and inflammatory indexes such as LDH, IL-6 and PLR could be useful in stratifying the risk for COVID-19 patients to develop VTE manifestations.

Unfortunately, there is still a gap of knowledge on the certain pathogenesis of VTE related to SARS-CoV-2 infection, so that we cannot exclude that a part of the PE reported were pulmonary thrombosis and not traditional isolated PE $[34,35]$. 


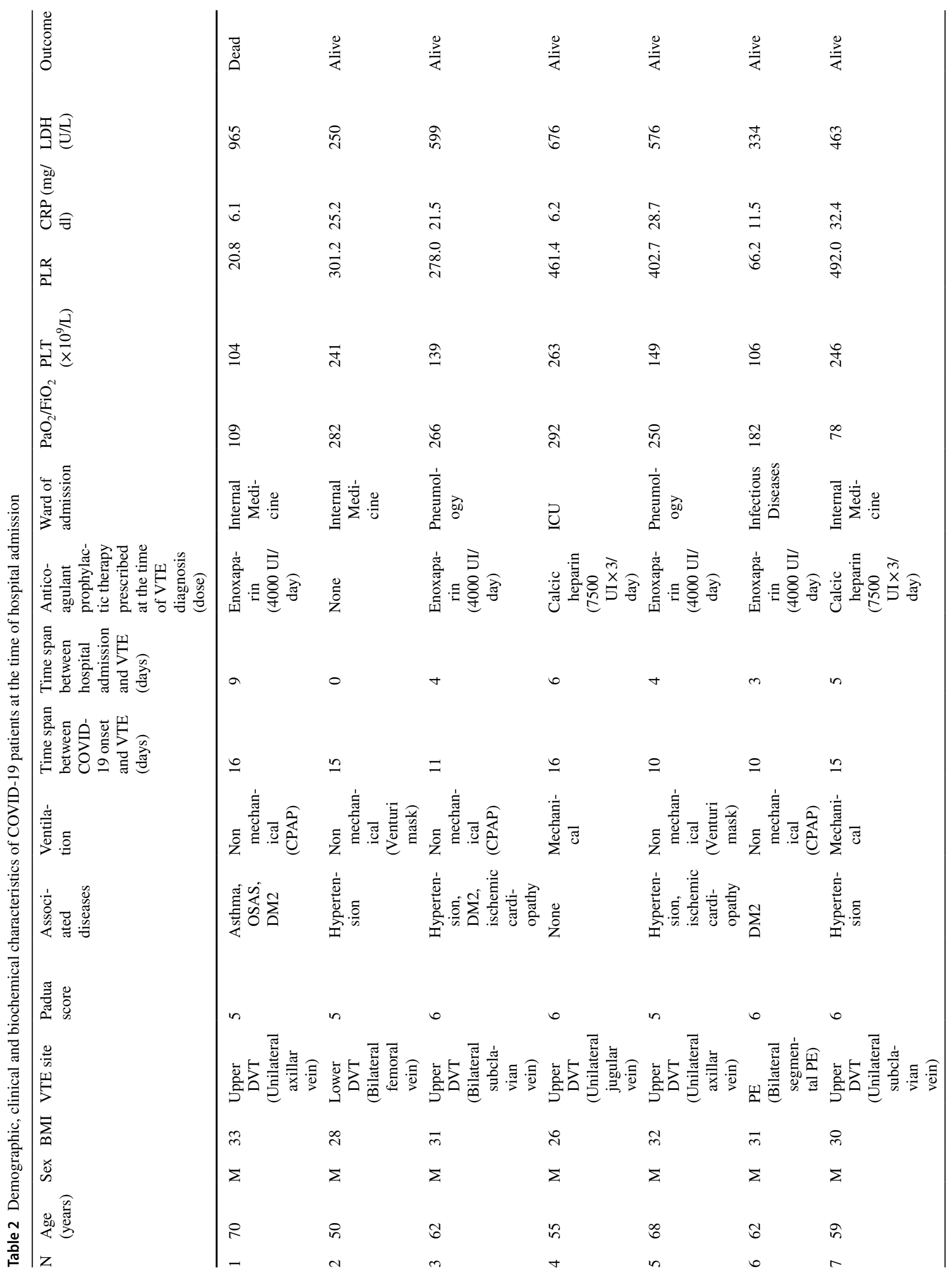




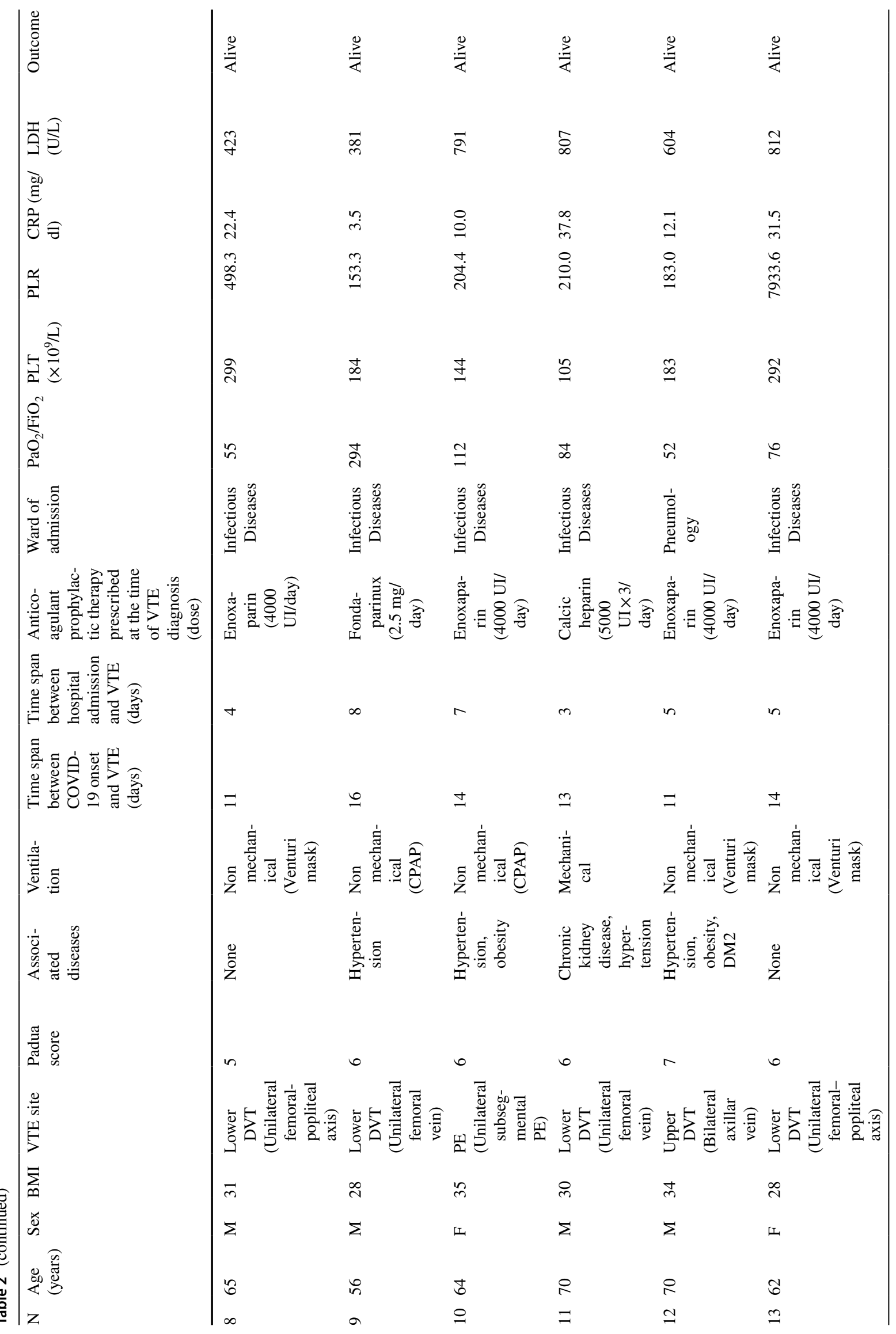




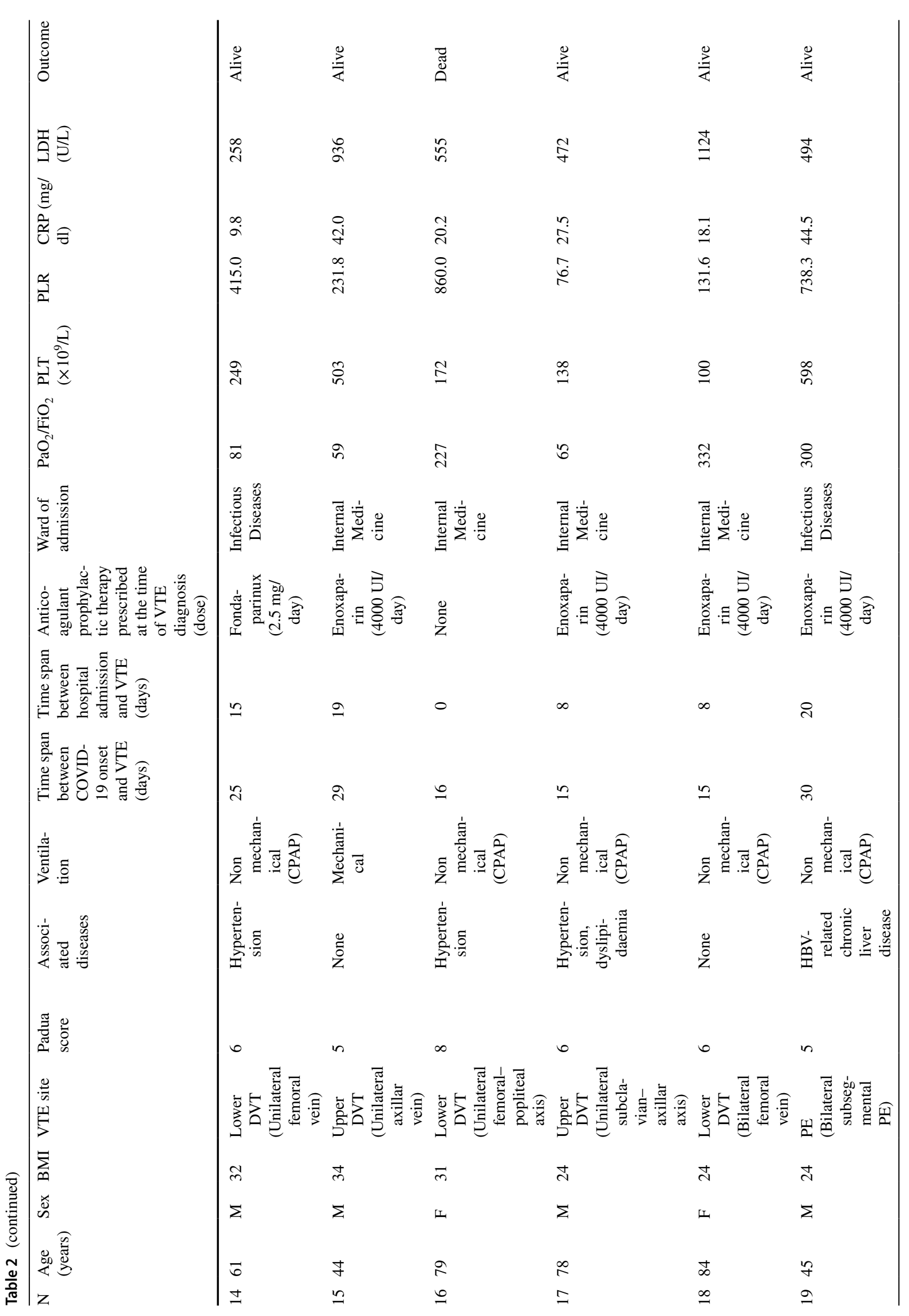




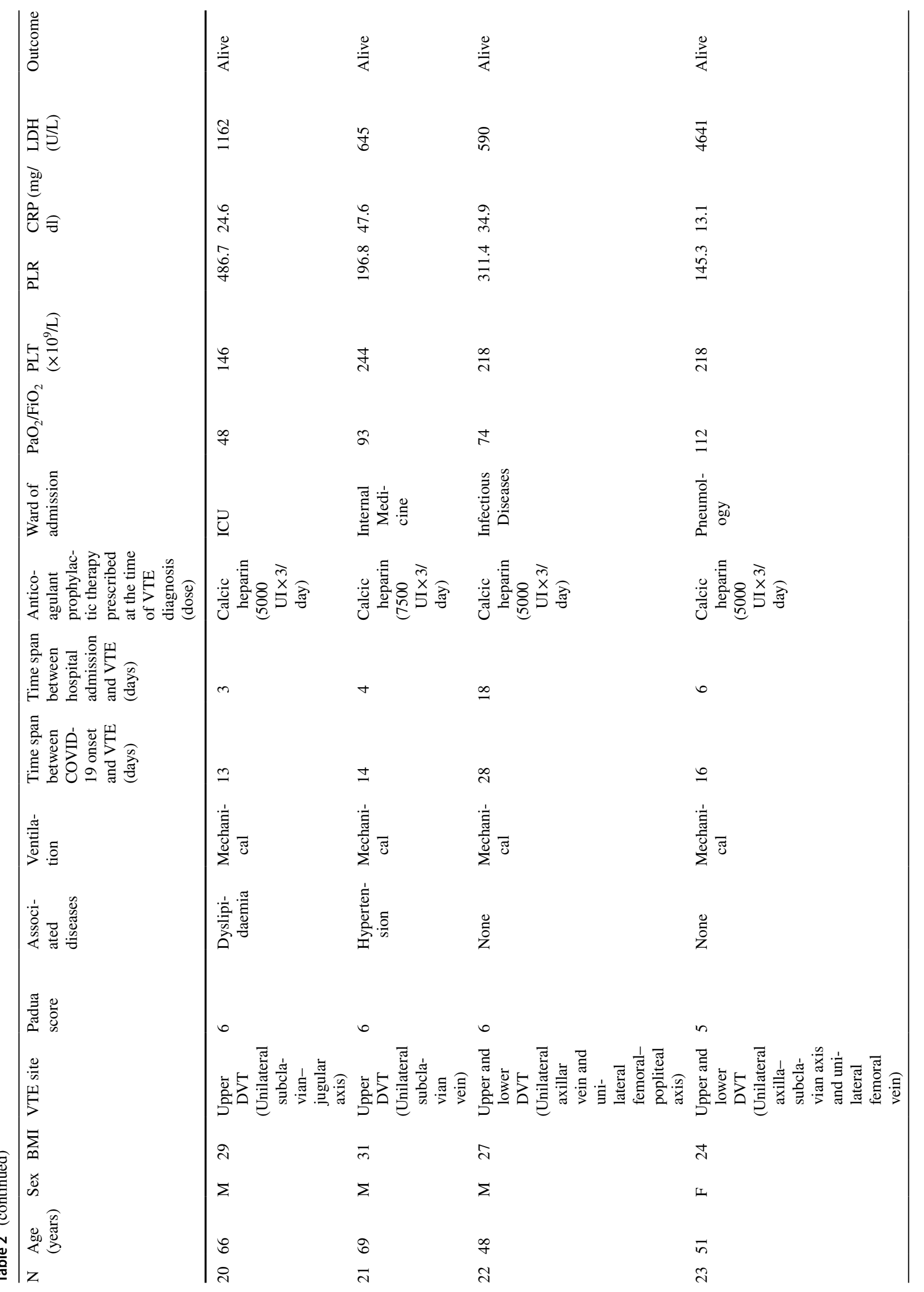




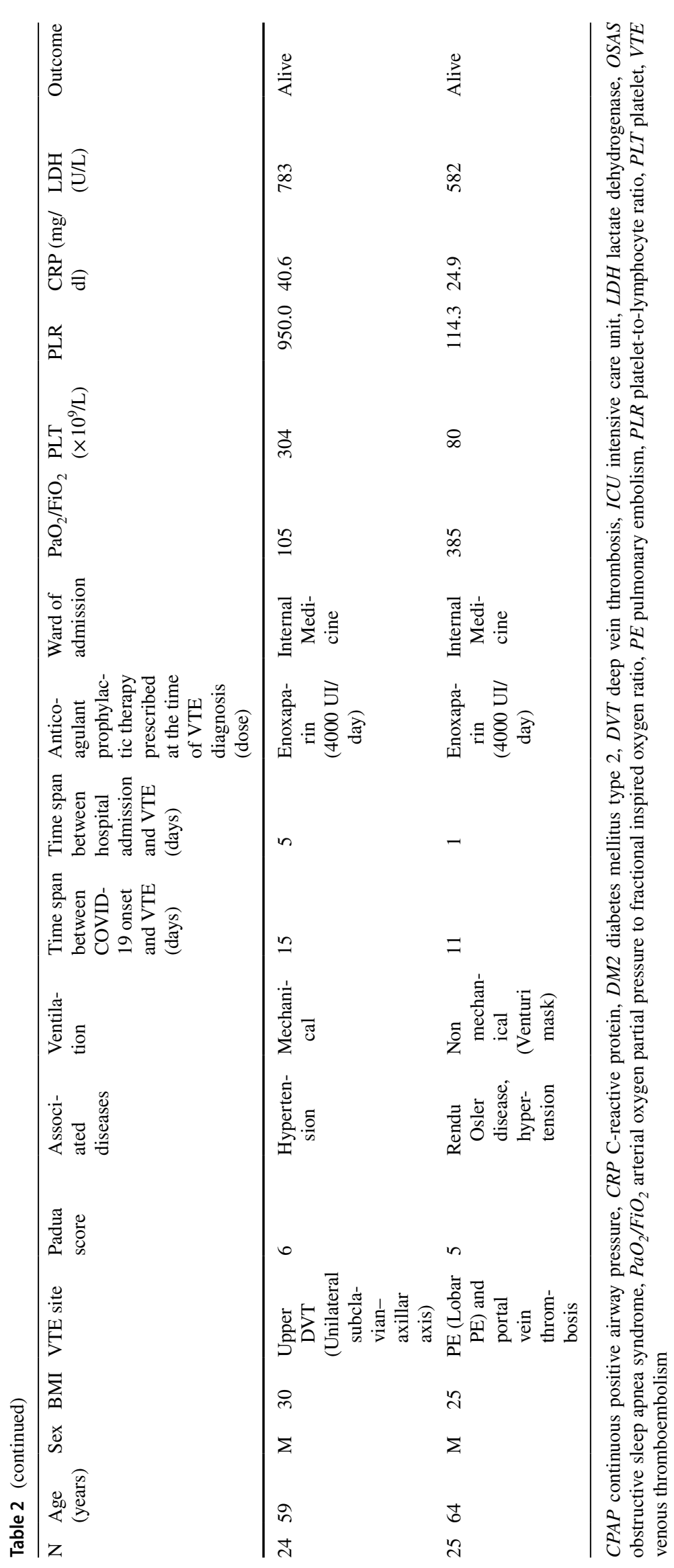


Indexes such as MPV and PLR have been used as unfavorable predictors in other viral infections [36, 37]. The existence of a significant correlation between PLR and inflammatory indexes in the VTE subcohort seems to support the role of this marker as an unfavorable predictor in COVID-19 patients, in keeping with recent reports [38].

In contrast with Tang et al. [39], we did not find any significant difference in D-dimer between the two cohorts and this could be related to the numerical imbalance between VTE and non VTE patients.

Our study also showed that, unexpectedly, platelet count was only slightly reduced or normal in most of COVID19 patients. That could be explained by the inflammatory state too, since thrombopoietin could increase in response to lung inflammation [40]. Hence, inflammatory profile, more than platelet count and coagulation profile, could help us to determine and estimate the severity of COVID-19. According to recent publications, we believe that enoxaparin and fondaparinux should be preferred instead of calcic heparin because of its anti-inflammatory properties [41]. Moreover, Mycroft-West et al. [42], showed that LMWH can bind to the SARS-CoV-2 surface protein (Spike) S1 receptor binding domain and block the replication of the virus, thus showing potential antiviral effects.

If early initiation of prophylactic anticoagulant therapy should be recommended in COVID-19 patients [8], how to optimize anticoagulant therapy is still a matter of debate [43]. It can be assumed that by implementing the dosage of LMWH traditionally considered as prophylactic, if renal function is permissive and in the absence of bleeding diathesis, most VTE events could be prevented, but further studies are necessary to confirm this therapeutic strategy.

Indeed, this study has some limitations, including the small sample size, the numerical imbalance between VTE and non-VTE cohorts, the lack of follow-up data, and the scarce use of chest CT-scan in the imaging diagnostic protocol of COVID-19 pneumonia at the time of hospital admission. Moreover, even if catheter-related DVTs were not included in the study, we cannot exclude that blood sampling could partially explain such a high incidence.

Nevertheless, our study confirmed the high rate of VTE in COVID-19 patients, especially in those admitted to the ICU. If anticoagulant therapy is then essential in the management of these patients, attention should be paid to the bleeding risk, due to the increased incidence of peptic ulcer disease in COVID-19 patients [44].

The relationship between VTE and inflammation might support the hypothesis that a cytokine burst plays a central role in thrombotic complication in COVID-19 patients. Supporting our speculation, recent works have discussed how hyperinflammation and detrimental immunothrombosis might play a central role in COVID-19 pathophysiology. The short median time lapse between hospital admission and VTE onset suggests that this phenomenon is not correlated with hospitalization, but rather with COVID-19 per se.

Since $100 \%$ of VTE patients had a Padua score greater than 4 , we suggest performing upper and lower limb venous ultrasound in COVID-19 patients with a score $>4$, as well as routinely in ICU patients to identify and treat DVT as soon as possible. We also suggest routine CT scans in patients with hypoxemia that is not fully explained by lung imaging, as well as in those with a profile risk similar to that described in the cohort of VTE patients.

Acknowledgements Dr. Federica Melazzini is grateful to the University of Pavia for supporting her research projects. We thank all the specialists, resident physicians, and all healthcare professionals who are currently facing the COVID-19 epidemic at San Matteo Hospital Foundation. We thank Dr. Lorenzo Neill Aldegheri for having proofread the manuscript.

San Matteo Pavia COVID-19 Task Force Internal Medicine Department Nicola Aronico, Gaetano Bergamaschi, Giampiera Bertolino, Silvia Codega, Filippo Costanzo, Roberto Cresci, Angela Delliponti, Giuseppe Derosa, Michele Di Stefano, Francesco Falaschi, Carmine Iadarola, Elisabetta Lovati, Pietro Carlo Lucotti, Alessandra Martignoni, Caterina Mengoli, Emanuela Miceli, Amedeo Mugellini, Chiara Muggia, Elisabetta Pagani, Ilaria Palumbo, Alessandro Pecci, Tiziano Perrone, Carmelo Sgarlata, Luisa Siciliani, Andrea Staniscia, Francesca Torello Vjera, Giovanna Achilli, Andrea Agostinelli, Valentina Antoci, Alessia Ballesio, Francesco Banfi, Chiara Barteselli, Irene Benedetti, Federica Borrelli de Andreis, Michele Brattoli, Francesca Calabretta, Ginevra Cambiè, Roberta Canta, Federico Conca, Luigi Coppola, Elisa Maria Cremonte, Gabriele Croce, Virginia Del Rio, Francesco Di Terlizzi, Maria Giovanna Ferrari, Sara Ferrari, Anna Fiengo, Tommaso Forni, Chiara Frigerio, Alessandra Fusco, Margherita Gabba, Matteo Garolfi, Antonella Gentile, Giulia Gori, Giacomo Grandi, Paolo Grimaldi, Alice Lampugnani, Francesco Lapia, Federica Lepore, Gianluca Lettieri, Jacopo Mambella, Chiara Mercanti, Stefania Merli, Francesco Mordà, Alba Nardone, Luca Pace, Lucia Padovini, Alessandro Parodi, Ivan Pellegrino, Lavinia Pitotti, Margherita Reduzzi, Giovanni Rigano, Giovanni Romito, Giorgio Rotola, Umberto Sabatini, Lucia Salvi, Giovanni Santacroce, Jessica Savioli, Simone Soriano, Carmine Spataro, Debora Stefani. Intensive Care Department Anna Rita Aliberti, Alessandro Amatu, Laura Anfossi, Eric Arisi, Chiara Baldi, Mirko Belliato, Lorenzo Bellini, Alberto Benzi, Germana Bichisao, Antonia Bolongaro, Andrea Bottazzi, Federica Broglia, Giacomo Bruschi, Luca Caneva, Emanuele Capaccio, Valeria Carboni, Fabrizio Cavalloro, Maria Ciceri, Luca Civardi, Maria Paola Delmonte, Elisa Lucia Domenegati, Federica Ferrari, Fiorenza Ferrari, Marta Ferrari, Marinella Fuardo, Maddalena Margherita Gerletti, Simonetta Gualdana, Marcella Ilardi, Claudia Lo Coco, Giuseppe Maggio, Maria Benedetta Mascia, Simonetta Mencherini, Paola Maria Merati, Silvia Mongodi, Anna Maria Mori, Federica Morgante, Thekla Larissa Niebel, Silvano Noli, Anita Orlando, Michele Pagani, Debora Passador, Simona Pellicori, Luciano Perotti, Raffaella Picchioni, Silvia Poma, Marco Pozzi, Emanuela Preti, Roberta Puce, Danila Katia Radolovich, Gianluca Ragni, Filippo Repossi, Francesca Riccardi, Roberto Rizzardi, Giuseppe Rodi, Emanuela Roldi, Giovanni Romito, Cristina Rossi, Giuseppe Sala Gallini, Fabio Sciutti, Debora Sportiello, Giulia Ticozzelli, Federico Visconti, Silvia Zizzi, Alessandro Bagliani, Corrado Belotti, Chiara Bossi, Andrea Colombo, Costanza Natalia Julia Colombo, Luca Cremascoli, Valentino Dammassa, Roberto Discepoli, Maria Adelaide Garlando, Filippo Grandini, Andrea Pellegrini, Cecilia Quaranta, Andrea Stella, Francesco Torresani. Infectious Disease Department Mario Mondelli, Enrico Brunetti, Angela Di Matteo, Elena Seminari, Laura Maiocchi, Valentina Zuccaro, Layla Pagnucco, Bianca 
Mariani, Serena Ludovisi, Raffaella Lissandrin, Aldo Parisi, Paolo Sacchi, Savino F. A. Patruno, Giuseppe Michelone, Roberto Gulminetti, Domenico Zanaboni, Stefano Novati, Renato Maserati, Paolo Orsolini, Marco Vecchia, Erika Asperges, Alessandro Di Filippo, Margherita Sambo, Simona Biscarini, Matteo Lupi, Silvia Roda, Ilaria Gallazzi, Michele Sachs, Pietro Valsecchi, Alessandra Ferrari, Bianca Mariani. Respiratory Disease Department Matteo Bosio, Alessandro Cascina, Valentina Conio, Rita Di Domenica, Anna Donnetta, Elia Fraolini, Giuseppe Gualtieri, Patrizia Mangiarotti, Francesca Mariani, Federica Meloni, Tiberio Oggionni, Lidia Pasturenzi, Vanessa Ronzoni, Laura Saracino, Giulia Stella, Stefano Tomaselli, Tommaso Abbate, Giulia Accordino, Francesco Bertuccio, Cecilia Burattini, Elisa Cacciatore, Elena Cattaneo, Vittorio Chino, Manuela Coretti, Matteo Della Zoppa, Cristina Infusino, Sara Lettieri, Valeria Maccabruni, Silvia Mancinelli, Claudio Tirelli, Valentina Vertui.

Author contributions All authors significantly participated in the drafting of the manuscript or critical revision of the manuscript for important intellectual content and provided approval of the final submitted version. Individual contributions are as follow: ADS, FM and MC designed and coordinated the study, interpreted data and wrote the manuscript. FB detected coronavirus in biologic specimens. All the other authors followed up patients, locally collected data, and reviewed the paper for final approval. ADS, FM, MVL and FM, reviewed the paper and made final critical revisions for important intellectual contents.

Funding Open access funding provided by Università degli Studi di Pavia within the CRUI-CARE Agreement.

\section{Compliance with ethical standards}

Conflict of interest The authors declare that they have no conflict of interest.

Ethical approval The study was performed as a clinical audit using routine collected clinical data. The study was approved by the local ethics committee (San Matteo Hospital Foundation) on March 13th 2020.

Human and animal rights statement All procedures performed in the study were in accordance with the ethical standards of the institutional and/or national research committee and with the 1964 Helsinki Declaration and its later amendments or comparable ethical standards.

Informed consent The study was performed as a clinical audit using routine collected clinical data in an anonymised format, and as such is exempt from the need to take specific written informed consent.

Open Access This article is licensed under a Creative Commons Attribution 4.0 International License, which permits use, sharing, adaptation, distribution and reproduction in any medium or format, as long as you give appropriate credit to the original author(s) and the source, provide a link to the Creative Commons licence, and indicate if changes were made. The images or other third party material in this article are included in the article's Creative Commons licence, unless indicated otherwise in a credit line to the material. If material is not included in the article's Creative Commons licence and your intended use is not permitted by statutory regulation or exceeds the permitted use, you will need to obtain permission directly from the copyright holder. To view a copy of this licence, visit http://creativecommons.org/licenses/by/4.0/.

\section{References}

1. Huang C, Wang Y, Li X et al (2020) Clinical features of patients infected with 2019 novel coronavirus in Wuhan, China. Lancet 395:497-506. https://doi.org/10.1016/S0140-6736(20)30183-5

2. Rodriguez-Morales AJ, Cardona-Ospina JA, Gutiérrez-Ocampo E et al (2020) Clinical, laboratory and imaging features of COVID19: a systematic review and meta-analysis. Travel Med Infect Dis. https://doi.org/10.1016/j.tmaid.2020.101623

3. Guan WJ, Ni ZY, Hu Y et al (2020) Clinical characteristics of coronavirus disease 2019 in China. N Engl J Med 382:1708-1720. https://doi.org/10.1056/NEJMoa2002032

4. Chen N, Zhou M, Dong X et al (2020) Epidemiological and clinical characteristics of 99 cases of 2019 novel coronavirus pneumonia in Wuhan, China: a descriptive study. Lancet 395:507-513. https://doi.org/10.1016/S0140-6736(20)30211-7

5. Li LQ, Huang T, Wang YQ et al (2020) 2019 novel coronavirus patients' clinical characteristics, discharge rate, and fatality rate of meta-analysis. J Med Virol. https://doi.org/10.1002/jmv.25757

6. Zhou F, Yu T, Du R et al (2020) Clinical course and risk factors for mortality of adult inpatients with COVID-19 in Wuhan, China: a retrospectivecohort study. Lancet 395(10229):1054-1062. https ://doi.org/10.1016/S0140-6736(20)30566-3

7. Cui S, Chen S, Li X, Liu S, Wang F (2020) Prevalence of venous thromboembolism in patients with severe novel coronavirus pneumonia. J Thromb Haemost. https://doi.org/10.1111/jth.14830

8. Marietta M, Ageno W, Artoni A et al (2020) COVID-19 and haemostasis: a position paper from Italian Society on Thrombosis and Haemostasis (SISET). Blood Transfus 18:167-169. https:// doi.org/10.2450/2020.0083-20

9. Zakai NA, McClure LA (2011) Racial differences in venous thromboembolism. J Thromb Haemost 9:1877-1882. https://doi. org/10.1161/CIRCULATIONAHA.113.006472

10. Klok FA, Kruip MJHA, Van der Meer NJM et al (2020) Incidence of thrombotic complications in critically ill ICU patients with COVID-19. Thromb Res. https://doi.org/10.1016/j.throm res.2020.04.013

11. Lodigiani C, Iapichino G, Carenzo L et al (2020) Venous and arterial thromboembolic complications in COVID-19 patients admitted to an academic hospital in Milan, Italy. Thromb Res 191:9-14. https://doi.org/10.1016/j.thromres.2020.04.024

12. Middeldorp S, Coppens M, van Haaps TF et al (2020) Incidence of venous thromboembolism in hospitalized patients with COVID-19. J Thromb Haemost. https://doi.org/10.1111/jth.14888

13. Llitjos JF, Leclerc M, Chochois $C$ et al (2020) High incidence of venous thromboembolic events in anticoagulated severe COVID19 patients. J Thromb Haemost. https://doi.org/10.1111/jth.14869

14. Helms J, Tacquard C, Severacet F et al (2020) High risk of thrombosis in patients in severe SARS-CoV-2 infection: a multicenter prospective cohort study. Intensive Care Med. https://doi. org/10.1007/s00134-020-06062-x

15. Poissy J, Goutay J, Caplan M et al (2020) Pulmonary embolism in COVID-19 patients: awareness of an increased prevalence. Circulation. https://doi.org/10.1161/CIRCULATIONAHA.120.047430

16. Barbar S, Noventa F, Rossetto V et al (2010) A risk assessment model for the identification of hospitalized medical patients at risk for venous thromboembolism: the Padua Prediction Score. J Thromb Haemost 8:2450-2457. https://doi.org/10.111 1/j.1538-7836.2010.04044.x

17. Von Elm E, Altman DG, Egger M et al (2007) The Strengthening the Reporting of Observational Studies in Epidemiology (STROBE) statement: guidelines for reporting observational studies. Ann Intern Med 147:573-577. https://doi.org/10.1016/j. jclinepi.2007.11.008 
18. Spyropoulos AC, Anderson FA Jr, FitzGerald G et al (2011) Predictive and associative models to identify hospitalized medical patients at risk for VTE. Chest 140:706-714. https://doi. org/10.1378/chest.10-1944

19. Hirsch DR, Ingenito EP, Goldhaber SZ (1995) Prevalence of deep venous thrombosis among patients in medical intensive care. JAMA. https://doi.org/10.1001/jama.1995.0353004006 3042

20. Minet C, Potton L, Bonadona A et al (2015) Venous thromboembolism in the ICU: main characteristics, diagnosis and thromboprophylaxis. https://doi.org/10.1186/s13054-015-1003-9

21. Gangireddy C, Rectenwald JR, Upchurch GR et al (2007) Risk factors and clinical impact of postoperative symptomatic thromboembolism. J Vasc Clin Surg 45:335-341. https://doi. org/10.1016/j.jvs.2006.10.034

22. Smeeth L, Cook C, Thomas S, Hall AJ, Hubbard R, Vallance P (2006) Risk of deep vein thrombosis and pulmonary embolism after acute infection in a community setting. Lancet 367:10751079. https://doi.org/10.1016/S0140-6736(06)68474-2

23. Esmon CT (2013) Molecular circuits in thrombosis and inflammation. Thromb Haemost 109:416-420. https://doi.org/10.1160/ TH12-08-0634

24. Von Bruhl ML, Stark K, Steinhart A et al (2012) Monocytes, neutrophils, and platelets cooperate to initiate and propagate venous thrombosis in mice in vivo. J Exp Med 209:819-835. https://doi.org/10.1084/jem.20112322

25. Fuchs TA, Brill A, Duerschmied D et al (2010) Extracellular DNA traps promote thrombosis. Proc Natl Acad Sci USA 107:15880-15885. https://doi.org/10.1073/pnas.1005743107

26. Massberg S, Grahl L, von Bruehl ML et al (2010) Reciprocal coupling of coagulation and innate immunity via neutrophil serine proteases. Nat Med 16:887-896. https://doi.org/10.1038/ nm. 2184

27. Hidalgo A, Chang J, Jang JE, Peired AJ, Chiang EY, Frenette PS (2009) Heterotypic interactions enabled by polarized neutrophil microdomains mediate thromboinflammatory injury. Nat Med 15:384-391. https://doi.org/10.1038/nm.1939

28. Jilma B, Marsik C, Kovar F, Wagner OF, Jilma-Stohlawetz P, Endler G (2005) The single nucleotide polymorphism Ser128Arg in the E-selectin gene is associated with enhanced coagulation during human endotoxemia. Blood 105:2380-2383. https://doi. org/10.1182/blood-2004-09-3752

29. De Vriese AS (2003) Prevention and treatment of acute renal failure in sepsis. J Am Soc Nephrol 14:792-805. https://doi. org/10.1097/01.ASN.0000055652.37763.F7

30. Alikhan R, Cohen AT, Combe S et al (2004) Risk factor for venous thromboembolism in hospitalized patient with acute medical illness: analysis of the MEDENOX Study. Arch Intern Med 164:963-968. https://doi.org/10.1001/archinte.164.9.963

31. Mukhopadhyay S, Johnson TA, Duru N et al (2019) Fibrinolysis and inflammation in venous thrombus resolution. Front Immunol 10:1348. https://doi.org/10.3389/fimmu.2019.01348
32. Marongiu F, Grandone E, Barcellona D (2020) Pulmonary thrombosis in 2019-nCoV pneumonia? J Thromb Haemost 18:1511. https://doi.org/10.1111/jth.14844

33. Dolhnikoff M, Duarte-Neto AN, de Almeida Monteiro RA et al (2020) Pathological evidence of pulmonary thrombotic phenomena in severe COVID-19. J Thromb Haemost 18:1517-1519. https ://doi.org/10.1111/jth.14844

34. Levi M (2018) Pathogenesis and diagnosis of disseminated intravascular coagulation. Int J Lab Hematol 40:15-20. https://doi. org/10.1111/ijlh.12830

35. Stroo I, Ding C, Novak A et al (2018) Inhibition of the extrinsic or intrinsic coagulation pathway during pneumonia-derived sepsis. Am J Physiol Lung Cell Mol Physiol 315:L799-L809. https://doi. org/10.1152/ajplung.00014.2018

36. Cai J, Wang K, Han T, Jiang H (2018) Evaluation of prognostic values of inflammation-based makers in patients with HBV-related acute-on-chronic liver failure. Medicine (Baltimore) 97:e13324. https://doi.org/10.1097/MD.0000000000013324

37. Fei Y, Zhang H, Zhang C (2019) The application of lymphocyte/ platelet and mean platelet volume/platelet ratio in influenza A infection in children. J Clin Lab Anal 33:e22995. https://doi. org/10.1002/jcla.22995

38. Yang AP, Liu JP, Tao WQ, Li HM (2020) The diagnostic and predictive role of NLR, d-NLR and PLR in COVID-19 patients. Int Immunopharmacol 84:106504. https://doi.org/10.1016/j.intim p.2020.106504

39. Tang N, Bai H, Chen X, Gong J, Li D, Sun Z (2020) Anticoagulant treatment is associated with decreased mortality in severe coronavirus disease 2019 patients with coagulopathy. J Thromb Haemost 8:1094-1099. https://doi.org/10.1111/jth.14817

40. Menter DG, Kopetz S, Hawk E et al (2017) Platelet "first responders" in wound response, cancer, and metastasis. Cancer Metastasis Rev 36:199-213. https://doi.org/10.1007/s10555-017-9682-0

41. Cassinelli G, Naggi A (2016) Old and new applications of nonanticoagulant heparin. Int J Cardiol. https://doi.org/10.1016/ S0167-5273(16)12004-2

42. Mycroft-West C, Su D, Elli S et al (2020) The 2019 coronavirus (SARS-CoV-2) 359 surface protein (Spike) S1 Receptor Binding Domain undergoes conformational change upon 360 heparin binding. BioRxiv. https://doi.org/10.1101/2020.04.29.068486

43. Thachil J (2020) The versatile heparin in COVID-19. J Thromb Haemost 18:1020-1022. https://doi.org/10.1111/jth.14821

44. Melazzini F, Lenti MV, Mauro A, De Grazia F, Di Sabatino A (2020) Peptic ulcer disease as a common cause of bleeding in patients with coronavirus disease 2019. Am J Gastroenterol. https ://doi.org/10.14309/ajg.0000000000000710

Publisher's Note Springer Nature remains neutral with regard to jurisdictional claims in published maps and institutional affiliations. 